\title{
Numerical Investigation of Quantum Chaos in the Problem of Multichannel Scattering in Three Body System
}

\author{
A.V. Bogdanov, A.S. Gevorkyan, and A.A. Udalov \\ Institute for High-Performance Computing and Data Bases \\ P/O Box 71, 194291, St-Petersburg, Russia, \\ bogdanov@hm.csa.ru, ashot@fn.csa.ru, udalov@fn.csa.ru.
}

\begin{abstract}
The first principle calculations of quantum chaos in the framework of representation constructed by authors for multi-channel quantum scattering was made. Based on intrinsic properties of scattering system the numerical task was divided into independent subtasks and the parallel algorithm for numerical computations was developed and tested on massive-parallel systems Parsytec CC/16 and SPP-1600. This algorithm made it possible to carry out converging computations for three-body problem for any energy. It was shown, that even in the simple case of three-body problem the principle of quantum determinism breaks down in general and one has a micro-irreversible quantum mechanics. The ab initio calculations of the quantum chaos (wave chaos) were carried out on the example of an elementary chemical reaction $L i+(F H) \rightarrow(L i F H)^{*} \rightarrow(L i F)+H$.
\end{abstract}

\section{Introduction}

At the early stage of quantum mechanics, Albert Einstein wrote the work in which the question was touched, which became a focus of physicists attention several decades later. The question was: what will the classic chaotic system become in terms of quantum mechanics. He has particularly set apart the threebody problem.

In a trial to understand the influence of chaotic dynamical features on quantum quantities calculation the numerical approach plays an important role. In this connection one needs to have high performance algorithms, which allow to make calculations with as small time costs as possible. Numerical results also give sometimes a heuristic ideas, which may be very useful in theoretical considerations.

For the first time the problem of quantum chaos was studied by the authors on the example of quantum multi-channel scattering in collinear three-body system [1,2]. It was shown that this case can be transformed into a problem of a forced unharmonic oscillator with non-trivial time (internal time).

In the present work we discuss the possibilities of chaotic phenomena calculations based on our approach. 


\section{Formulation of the Problem}

The quantum multi-channel scattering in the framework of collinear model is considered. As it was shown elsewhere 12 the problem of quantum evolution in this case can be strictly formulated as an image point with a mass $\mu_{0}$ moving over the manifold $M$, the latter being a stratificated Lagrange surface $S_{p}$. At that the motion is related to the local coordinate system moving over $S_{p}$. In our case there is standard definition of the surface $S_{p}$

$$
S_{p}=\left\{x^{1}, x^{2} ; P^{2}\left(x^{1}, x^{2}\right)>0\right\}, \quad P^{2}\left(x^{1}, x^{2}\right)=2 \mu_{0}\left(E-V\left(x^{1}, x^{2}\right)\right),
$$

$E$ and $V\left(x^{1}, x^{2}\right)$ being the total energy and interaction potential of the system respectively. The metric on the surface $S_{p}$ in our case is introduced in the following way: $g_{i k}=P^{2}\left(x^{1}, x^{2}\right) \delta_{i k}$.

The motion of the local coordinate system, it is determined by the projection of the image point moving along the extremal ray $\Im_{\text {ext }}$ of the Lagrange manifold $S_{p}$. Note, that for scattering problem under consideration there are two extremal rays on the surface $S_{p}$ : the one corresponding to particle rearrangement and the other corresponding to three free particles. In this paper we study only the first case, namely the one of rearrangement. The quantum evolution of the system on the manifold $M$ is described by the equation (see [2])

$$
\left\{\hbar^{2} \Delta_{\left(x^{1}(s), x^{2}\right)}+P^{2}\left(x^{1}(s), x^{2}\right)\right\} \Psi=0,
$$

with the operator $\Delta_{\left(x^{1}(s), x^{2}\right)}$ determined in curvilinear coordinates $\left(x^{1}, x^{2}\right)$ in Euclidean space $R^{2}, x^{1}(s)$ being the $x^{1}$-coordinate of image point trajectory. The metric tensor of the manifold $M$ is presented in [2]. Note, that the main difference of (2) from Schrödinger equation comes from the fact, that one independent coordinate, namely, $x^{1}(s)$ is derived from the set of nonlinear differential equations and is not a natural parameter of the problem. In certain situations it can be a chaotic function.

Our purpose is to find a solution of equation (2) that satisfies the following asymptotic conditions for the total wave function of the system

$$
\begin{gathered}
\lim _{\left(s, x^{1}\right) \rightarrow-\infty} \Psi^{(+)}\left(x^{1}(s), x^{2}\right)=\Psi_{\text {in }}\left(n ; x^{1}, x^{2}\right)+\sum_{m \neq n} R_{m n} \Psi_{i n}\left(m ; x^{1}, x^{2}\right), \\
\lim _{\left(s, x^{1}\right) \rightarrow+\infty} \Psi^{(+)}\left(x^{1}(s), x^{2}\right)=\sum_{m} S_{m n} \Psi_{\text {out }}\left(m ; x^{1}, x^{2}\right),
\end{gathered}
$$

where the coefficients $R_{m n}$ and $S_{m n}$ are the excitation and rearrangement amplitudes respectively.

\section{Solution of Schrödinger Equation on Manyfold $M(\Im(u))$}

Taking into account the fact that scattering wave function is located along the reaction coordinate $\Im$ and using the parabolic equation method [3] for such a 
problem, we represent the solution of (2) in a form

$$
\Psi^{(+)}\left(x^{1}(s), x^{2}\right)=\exp \left(i \hbar^{-1} \int_{0}^{x^{1}(s)} p\left(x^{1}\right) \sqrt{\gamma_{0}} d x^{1}\right) A\left(x^{1}(s), x^{2}\right),
$$

where $p\left(x^{1}\right)=P\left(x^{1}, 0\right)$ and $\gamma_{0}$ depends on metric on $S_{p}$. After the coordinate transformation in equation (2)

$$
\tau=(E)^{-1} \int_{0}^{x^{1}(s)} p\left(x^{1}\right) \sqrt{\gamma_{0}} d x^{1}, \quad z=(\hbar E)^{-1 / 2} p\left(x^{1}(s)\right) x^{2},
$$

one gets for the total wave function of three-body system in harmonic approximation [2]

$$
\widetilde{\Psi}^{(+)}(n ; z, \tau)=\left[\frac{\left(\Omega_{i n} / \pi\right)^{\frac{1}{2}}}{2^{n} n !|\xi|}\right]^{\frac{1}{2}} \exp \left[i \hbar^{-1} S_{e f f}(z, \tau)\right] H_{n}\left[\frac{\sqrt{\Omega_{i n}}}{|\xi|}(z-\eta)\right]
$$

where

$$
\begin{gathered}
S_{\text {eff }}(z, \tau)=S_{c l}(\tau)-E_{v}^{i} \int_{0}^{\tau}|\xi|^{-2} d \tau^{\prime}+ \\
+\left\{\dot{\eta}(z-\eta)+\frac{1}{2} \dot{\xi} \xi^{-1}(z-\eta)^{2}-\frac{1}{2} \dot{p} p^{-1} z^{2}\right\}, \\
S_{c l}(\tau)=E \tau-E \int_{-\infty}^{\tau}\left\{\frac{1}{2}\left[(\dot{\eta})^{2}-\Omega^{2}\left(\tau^{\prime}\right) \eta^{2}\right]+F\left(\tau^{\prime}\right) \eta\right\} d \tau^{\prime}, \\
E_{v}^{i}=\hbar \Omega_{\text {in }}\left(n+\frac{1}{2}\right), \quad \Omega_{\text {in (out })}=\lim _{\tau \rightarrow \pm \infty} \Omega(\tau) .
\end{gathered}
$$

The functions $\Omega^{2}(\tau)$ and $F(\tau)$ are defined on $S_{p}$ and are known, function $H_{n}(x)$ is a Hermitian polynomial. The function $\xi(\tau)$ is the solution of classical oscillator problem with usual scattering asymptotic condition. As to the function $\eta(\tau)$ it is expressed in terms of $\xi(\tau)$ (see [2]).

\section{Transition Amplitude for Rearrangement Processes}

One can show that transition probabilities for the reaction $A+(B, C)_{n} \rightarrow$ $(A B C)^{*} \rightarrow(A, B)_{m}+C$ have the form

$$
W_{m n}=\left|S_{m n}\right|^{2}=\frac{(1-\theta)^{1 / 2}}{m ! n !}\left|H_{m n}\left(b_{1}, b_{2}\right)\right|^{2} \exp [-\nu(1-\sqrt{\theta} \cos 2 \varnothing)],
$$

where the function $H_{m n}\left(b_{1}, b_{2}\right)$ is a complex Hermitian polynomial, and

$$
\begin{gathered}
b_{1}=\sqrt{\nu(1-\theta)} \exp (i \varnothing), \quad b_{2}=-\sqrt{\nu}[\exp (-i \varnothing)-\sqrt{\theta} \exp (i \varnothing)], \\
\varnothing=\frac{1}{2}\left(\delta_{1}+\delta_{2}\right)-\beta .
\end{gathered}
$$


Denoting $c=\left(\Omega_{\text {in }} / \Omega_{\text {out }}\right)^{1 / 2}$ one has for $\theta, \delta_{1}, \delta_{2}, \beta$ and $\nu$

$$
\begin{gathered}
c_{1}=e^{i \delta_{1}} c(1-\theta)^{-1 / 2}, \quad c_{2}=e^{i \delta_{2}} c[\theta /(1-\theta)]^{1 / 2} \\
\theta=\left|c_{2} / c_{1}\right|^{2}, \quad d=\lim _{\tau \rightarrow+\infty} d(\tau)=\sqrt{\nu} \exp (i \beta) .
\end{gathered}
$$

where $d(\tau)=\left(2 \Omega_{i n}\right)^{-1 / 2} \int_{-\infty}^{\tau} d \tau^{\prime} \xi\left(\tau^{\prime}\right) F\left(\tau^{\prime}\right)$ and the constants $c_{1}$ and $c_{2}$ enter into an asymptotic expression for $\xi(\tau)$ in the limit $\tau \rightarrow \pm \infty$ (see [2]).

\section{Numerical Calculations}

Schematically algorithm of numerical calculations may be described as a sequence of stages:

- I - Lagrange surface construction for the system. The curvilinear coordinate system, within which all further calculations are performed, is introduced on it;

- II - classical trajectory problem solution. At this stage the set of four ordinary non-linear differential equations of the first order is solved numerically. Essential initial parameters are collision energy $E$ and oscillator quantum number $n$. The set is solved by one-step method of $4^{t h}-5^{t h}$ order. This method is conditionally stable (by deviation of initial data and rhs) [4, therefore the standard automatic step decreasing method is implied to provide its stability. It's worth mentioning that initial set is degenerate in certain points. To eliminate these the standard $\sigma$-procedure with differentiation parameter replacement is performed.

- III - the results of classical trajectories calculations are used for calculations of complete quantum wave function in its final state. At this stage, the numerical problem consists in solution of an ordinary non-linear second order differential equation. Numerical investigation of this equation is a difficult task due to non-trivial behavior of differentiation parameter. Differentiation algorithm consists of two stages: 1) construction of differentiation parameter values grid using the results of classical problem calculation and 2) integration of initial differential equation on obtained non-uniform grid by means of multi-step method. Integration stability is provided by selection the integration step in a classical problem, control being performed by means of step-by-step truncation error calculation [4]. The obtained solution of differential equation is approximated in a final asymptotic state in a form of incoming and reflected flat waves superposition;

- IV - the results of quantum problem solution are used for obtaining the values of transition probabilities matrix elements and corresponding crosssections. Calculation of matrix elements for initial oscillator quantum number $n$ and final oscillator quantum number $m$ is performed with the use of expressions presented in [5]. Let's note that transition probability matrix obtained corresponds to one value of collision energy, stipulated at stage II; 
Let us remind that calculations for steps II and III are made for specific values of collision energy $E$ and oscillator quantum number of initial state $n$. Results of these calculations allow to obtain one vector of a reaction cross-section matrix, which corresponds to $n$. In order to obtain the entire cross-section matrix, calculations at stages II and III need to be repeated as many times as dictated by the size of reaction cross-section matrix. As a result the entire probability matrix is obtained. The procedure described needs to be repeated for many values of collision energy $E$ in order to enable further integration and velocity constants finding.

Algorithm of numerical calculations allows to perform the parallelization and use the multiprocessor supercomputers with massive parallel architecture for calculations.

Further we will show how the algorithm presented can be parallelized for massive parallel supercomputers with distributed and shared memory.

- Calculation algorithm for massive parallel systems with distributed memory. Calculation parallelization procedure is performed by the values of collision energy. Calculation of classical trajectory problem, quantum calculation and transition probability matrix calculation are performed in each of the parallel branches. Let's note that just as in the case on non-parallelized algorithm all calculations from stages II and III are performed as many times as it is dictated by the size of transition probability matrix. Due to the fact that calculation in each of thee parallel branches represents a separate problem and does not interact with other branches of calculation, the effectiveness of this parallelization algorithm using vs. relatively unparallelized algorithm is nearly proportional to a number of calculation branches, i.e. to the number of computation nodes.

This algorithm realization was performed on Parsytec $C C / 16$ supercomputer with massive parallel architecture with distributed memory. As a reaction on which the algorithm was tested, a well studied bimolecular reaction $L i+$ $(F H) \rightarrow(L i F H)^{*} \rightarrow(L i F)+H$ was taken. The results of testing have shown the calculation efficiency to be nearly proportional to the number of computation nodes.

- Calculation algorithm for massive parallel systems with shared memory. Just as in the previous algorithm, the first level of parallelization represents the distribution of calculations among the computation nodes in accordance with the values of collision energy. But, as can be seen from a scheme, in each of the parallel branches there is one more parallelization by the values of oscillator quantum number of the initial state as well. The second parallelization is based upon a fact that for classical trajectory problem calculation the same coefficients, that calculated "on-line", are used for different quantum numbers, thus allowing to make such a parallelization.

This algorithm was realized on $S P P-1600$ supercomputer with massive parallel architecture with shared memory. The results of testing have shown that just as it was expected, the efficiency of calculations is higher than in the previous example. 
a)

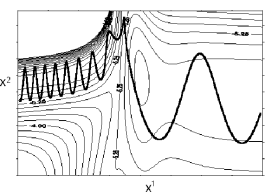

b)

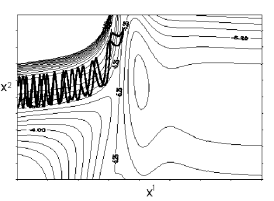

c)

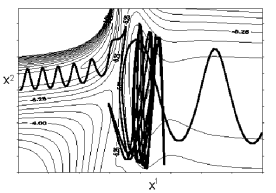

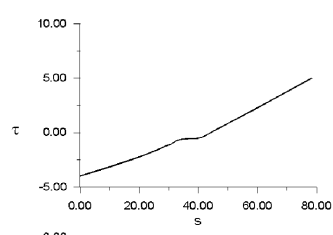
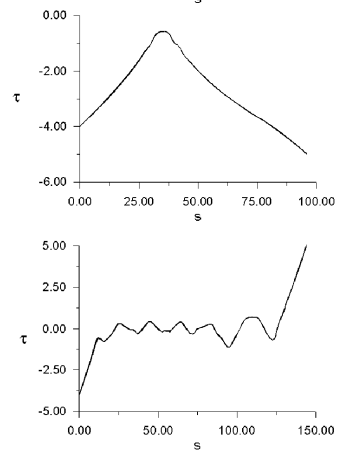

Fig. 1. Geodesic trajectories and internal time dependence on natural parameter $s$ for: a) - direct rearrangement process b) - direct reflection process and c) - rearrangement process going through the resonant state.

Finally we would like to stress one of the important features of parallelization algorithms demonstrated - their scalability. Due to the fact that integration of transition probability matrix and rate constants calculation during stage $\mathbf{V}$ requires the values of matrix elements for large number of energy values, one can hardly find a supercomputer with an excessive number of computation nodes.

\section{Investigation of Classical Trajectory Problem}

Numerical calculations were made for the reaction $L i+(F H) \rightarrow(L i F H)^{*} \rightarrow$ $(L i F)+H$. The potential surface for this reaction was reproduced using the quantum-mechanical calculations carried out in work [6]. Investigation of trajectory problem shows, that, starting from some energy values, internal time $\tau(s)$ from (5), which acts as a chronology parameter in the description of movement along a trajectory, becomes a nontrivial function, having an intricate dependence Fig 1 on the natural parameter (usual time). However, even if $\tau(s)$ is a natural parameter in some region, its derivative $\dot{\tau}(s)$ may have an irregular behavior (see Fig (2). The last property provides sufficient evidence for dynamical chaos existing in the system. As one can see from the Fig 3 the distribution of passed through into the subspace $R_{\text {out }}^{2}$ and reflected back into the subspace $R_{\text {in }}^{2}$ geodesic trajectories with respect to $x_{0}^{1}$ and $E$ for fixed main quantum number $n$ has irregular regions. One gets qualitatively the same picture for other values of $n$ too. Numerical calculations show, that for initial values regions mentioned above 
(a)
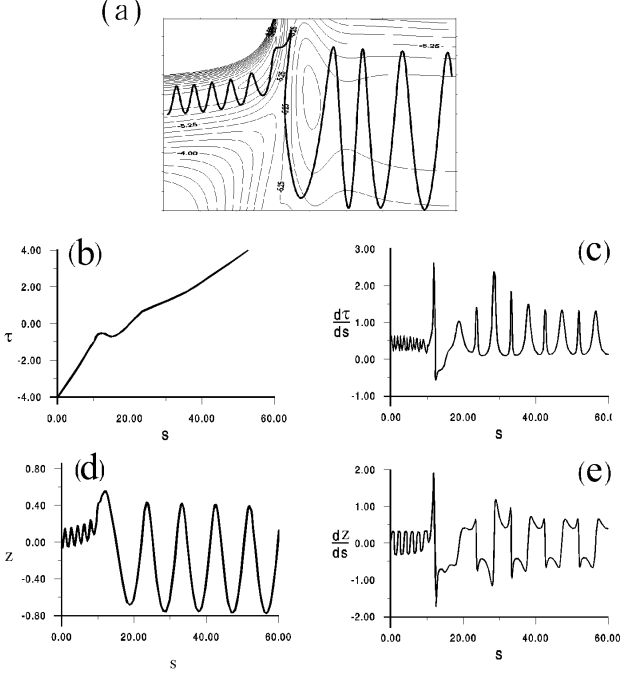

Fig. 2. a) - Geodesic trajectory characterizing direct reaction, b) - corresponding internal time, which is a natural parameter in a wide range, i.e. it is in one-to-one correspondence with parameter $s, \mathrm{c}$ ) - internal time derivative with respect to $s$, which has an irregular behavior, d) and e) - show transverse coordinate and its derivative with respect to $s$ respectively.

the main Lyapunov exponent is positive and grows fast, the last fact pointing to exponential divergence of geodesic trajectories. One can see from the results of calculations that the structure of chaotic behavior region is self-similar with respect to scale transformation Fig 3 . Chaotic behavior in the classical three body problem disappears when the total energy increases. Note that geometrical analysis of Lagrange manifold for reacting system $L i+F H$ also shows on the possibility of quasicompact submanifold existence, on which intermixing of trajectories may take place.

Similar calculations made for reacting system $\mathrm{N}_{2}+\mathrm{O}, \mathrm{N}_{2}+\mathrm{N}, \mathrm{N}+\mathrm{O}_{2}$, $\mathrm{N}+\mathrm{O}_{2}, \mathrm{O}_{2}+\mathrm{O}$ show the absence of any irregular regions on the map of passed through and reflected trajectories.

\section{Transition Probabilities Calculation for Rearrangement Process}

Let us consider the influence of irregular chaotic behavior of classical problem on quantum transition probabilities. It may be illustrated by Fig $4 \mathrm{a}$ and Fig $4 \mathrm{~b}$ which show the dependence of over-barrier transition probabilities in $L i+F H$ system on collision energy $E_{k}^{i}$ for fixed phases and quantum numbers. One can 


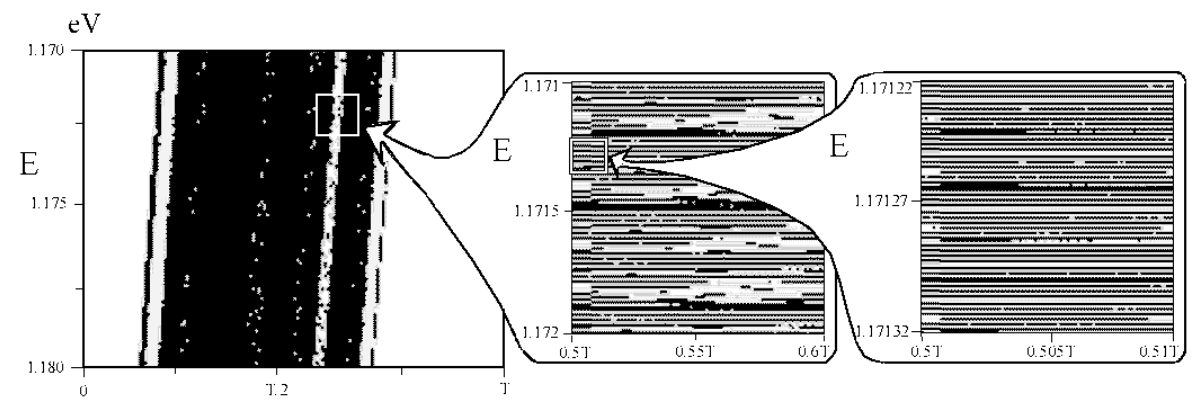

Fig. 3. Irregular map of initial values of the total energy $E$ and initial phase $x_{0}^{1}$ for passed through (white rectangles) and reflected back (black rectangles) geodesic trajectories. $T$ denotes a period of $x_{0}^{1}$.

see, that a small change in initial phase significantly changes the dependencies. In this connection the difficult problem arises to find the measure for the space (map) of passed through and reflected back geodesic trajectories. To calculate

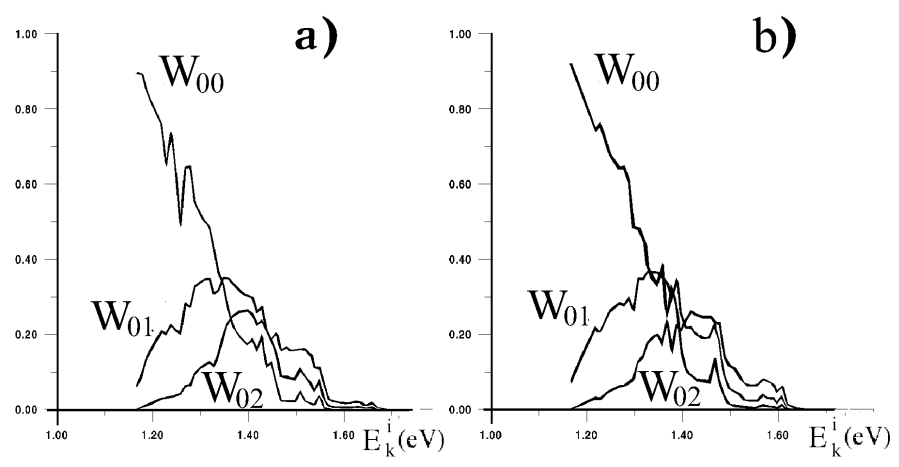

Fig. 4. (a) - Dependencies of transition probabilities $W_{00}, W_{01}$ and $W_{02}$ on collision energy $E_{k}^{i}$ for fixed phase $x_{0 i}^{1}$, (b) - the same dependencies, but calculated for the other (slightly differing from the first one) fixed phase $\bar{x}_{0 i}^{1}:\left|\bar{x}_{0 i}^{1}-x_{0 i}^{1}\right|=10^{-5} x_{0 i}^{1}$.

the total mean, giving the final probability for specific quantum transition as depending on energy, one have to average the corresponding quantum probability with respect to $\left(E_{k}^{i}, x_{0}^{1}\right)$ within the range $\left[\Delta E, \Delta x_{0}^{1}\right]$, where $\Delta E$ is a small interval of energies near $E_{k}^{i}$ and $\Delta x_{0}^{1}$ is a period of initial phase. The average process consists in that square $\Delta E \times \Delta x_{0}^{1}$ is divided on $n \gg 1$ rectangles, each of them having some phase point $x_{0 i}^{1}$ inside. Then each rectangle is subdivided by the grid with $M_{i}=l_{i} \times k_{i}$ nodes, $l_{i}$ and $k_{i}$ being the number of breaking points for $\Delta E$ 
and $\Delta x_{0}^{1} / n$ intervals respectively. Probability for geodesic trajectory (bearing ray) to pass through the $i$-th rectangle is calculated by the formula

$$
P\left(x_{i}^{1}, E_{k}^{i}\right)=\lim _{k_{i}, l_{i} \rightarrow \infty}\left(\frac{N_{i}}{M_{i}}\right),
$$

where $N_{i}$ counts how many times the bearing ray passes through into $R_{\text {out }}^{2}$ subspace. Weighted-mean probability is then calculated as the sum

$$
\Delta_{n m}(E)=\lim _{n \rightarrow \infty}\left\{\frac{1}{n} \sum_{i=1}^{n} P\left(x_{i}^{1}, E\right) W_{n m}\left(x_{i}^{1}, E\right)\right\} .
$$

After the averaging with use of (12) the dependence of transition probabilities on collision energy $E_{k}^{i}$ for $L i+F H \rightarrow L i F+H$ reaction becomes smooth, as one can see from Fig 5

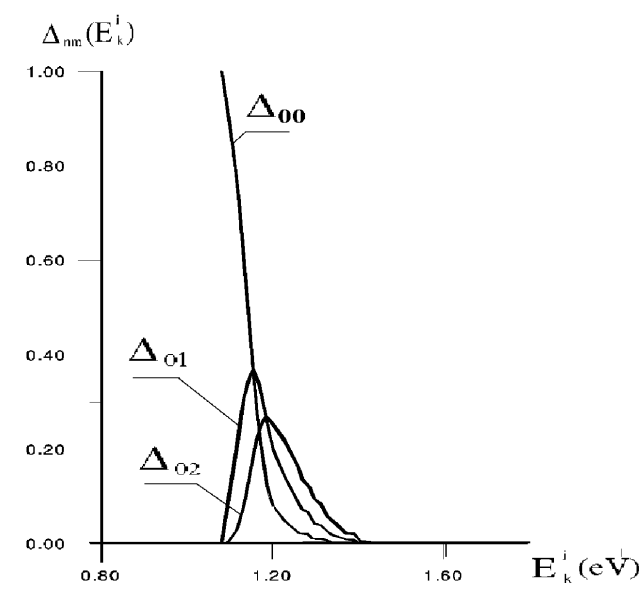

Fig. 5. Transition probabilities dependencies after averaging with respect on phase.

\section{Conclusion}

The reduction of multi-channel scattering problem solution to trajectory problem allows to use up-to-date multiprocessor computers with massive-parallel architecture and to calculate more trajectories within short period of time. In particular, about $10^{5}$ trajectories were calculated for energy range, within which the resonant state arising is possible. It was shown numerically, that an interval of energies exists in which the internal time dependence on the natural parameter 
$s$ may be of oscillatory type. At a small decrease of collision energy, a number of internal time oscillations grows dramatically. In this case the system loses all the information about its initial state completely. Chaos arises in a wave function, which then organizes itself into a new order within the limit $\tau \rightarrow \infty$. Mathematically it becomes possible as a result of common wave equation irreversibility by time. One of the numerical results of this work is a chaos map construction Fig 3. There was also numerically shown a strong sensitivity (for chaotic conditions) of quantum transition probabilities dependencies on energy to small changes of initial phase. It was shown that statistical approach must be used to calculate smooth dependencies of probabilities on energy, and the formula (12) for calculation was presented. Let's stress that the result obtained supports the transitional complex theory, developed by Eyring and Polanyi on the basis of heuristic considerations, the essence of the method being statistical description of chemical reactions.

\section{References}

[1] A. V. Bogdanov, A. S. Gevorkyan, Three-body multichannel scattering as a model of irreversible quantum mechanics, Proceedings of the International Symposium on Nonlinear Theory and its Applications, Hilton Hawaiian Village, 1997, V.2, pp.693-696.

[2] A.V.Bogdanov, A.S.Gevorkyan A.G.Grigoryan, S.A.Matveev, Internal time peculiarities as a cause of bifurcations arising in classical trajectory problem and quantum chaos creation in three-body system, Int. Journ. Bifurcation and Chaos, v. 9, N. 12 , p. $9-15,1999$.

[3] V.M.Babich, V.S.Buldyrev, Asimptotic methods in short waves difraction theory, Nauka, 1972 [Russian].

[4] A. A. Samarskiy, Introduction to the Numerical Methods (in russian), "Nauka", Moscow, (1997).

[5] A. N. Baz', Ya. B. Zel'dovich and A. M. Perelomov, Scattering reactions and Decays in Nonrelativistic Quantum Mechanics (in russia), "Nauka", Moscow, (1971).

[6] S. Carter, J. N. Murrell Analytical Potentials for Triatomic Molecules, Molecular Physics, v. 41, N. 3, pp. 567-581, (1980). 